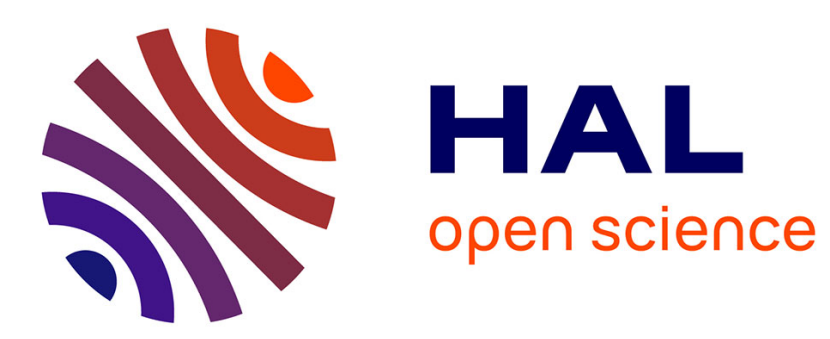

\title{
A Fault Localization Method for Single-phaseto Ground Faults in LV Smart Distribution Grids
}

Nikolaos Sapountzoglou, Bertrand Raison, Nuno Silva

\section{To cite this version:}

Nikolaos Sapountzoglou, Bertrand Raison, Nuno Silva. A Fault Localization Method for Singlephaseto Ground Faults in LV Smart Distribution Grids. This is a pre-print of a contribution published in ELECTRIMACS 2019, May 2019, Salerno, Italy. pp.21 - 23. hal-02179160

\section{HAL Id: hal-02179160 \\ https://hal.science/hal-02179160}

Submitted on 17 Jul 2019

HAL is a multi-disciplinary open access archive for the deposit and dissemination of scientific research documents, whether they are published or not. The documents may come from teaching and research institutions in France or abroad, or from public or private research centers.
L'archive ouverte pluridisciplinaire $\mathbf{H A L}$, est destinée au dépôt et à la diffusion de documents scientifiques de niveau recherche, publiés ou non, émanant des établissements d'enseignement et de recherche français ou étrangers, des laboratoires publics ou privés. 


\title{
A Fault Localization Method for Single-phase to Ground Faults in LV Smart Distribution Grids
}

\author{
Nikolaos Sapountzoglou · Bertrand Raison · Nuno Silva
}

\begin{abstract}
A fault localization method for single-phase to ground short-circuit (SC) faults in low voltage (LV) smart distribution grids is presented in this paper. Both the use of rms voltage phase measurements and an analysis of symmetrical components of the voltage were investigated and compared in this study. Phase measurements were found to be more suitable for single-phase to ground faults. The described method is a three-step process beginning with the identification of the faulty branch, followed by the localization of the sector in which the fault occurred and concluding with the estimation of the fault distance from the beginning of the feeder. Fault resistance values of $0.1,1,5,10,50$, 100,500 and $1000 \Omega$ were tested. An heterogeneity analysis was performed to test the effect of the use of various conductors on the method. Faults in all three phases were implemented and simulated on a real-case of a semi-rural LV distribution network of Portugal, provided by Efacec. Finally, the method presented an average estimation accuracy of $89.33 \%$ and an increased accuracy of $93.11 \%$ for low impedance faults (up to $10 \Omega$ of fault resistance).
\end{abstract}

\section{Introduction}

Electricity interruptions have huge economic and social impact. Production loss, restart costs, equipment damage and

Nikolaos Sapountzoglou $\cdot$ Bertrand Raison

Univ. Grenoble Alpes, CNRS, Grenoble INP*, G2Elab

38000 Grenoble, France

e-mail: nikolaos.sapountzoglou@g2elab.grenoble-inp.fr,

bertrand.raison@g2elab.grenoble-inp.fr

Nuno Silva

Efacec

Maia, Portugal

e-mail:nuno.silva@efacec.com

* Institute of Engineering Univ. Grenoble Alpes raw materials spoilage can be very costly. At the same time, uncomfortable temperatures at work or home, loss of leisure time and risk to health and safety (e.g. interrupting hospital service or industrial operations) are some of the aspects of electricity interruptions societal impact [1].

Faults that appear in distribution networks are responsible for the majority of customer interruptions [2]. Weather conditions (hurricane, lightning, etc.), component wearing and accidents are some of the causes of such faults. Two indices are used: a) the system average interruption duration index (SAIDI) and b) the Value of Lost Load (VoLL), to measure the impact of electricity interruptions in terms of time and cost repsectively. A maximum SAIDI of $371 \mathrm{~min}$ was noticed in Europe in 2016 [3]. Additionally, according to [4], the annual average of VoLL in Europe in 2013 was $8.37 € / k W h$.

For the aforementioned reasons, a fault should be detected, located and isolated as quickly as possible. All three of them are key elements of a self-healing smart grid and they include the following steps:

a) detecting the fault usually through threshold crossing detection techniques,

b) localizing the fault with any of the five most popular methods [5]: impedance based which are the most widely used [6-8], knowledge based [9-11], traveling wave [12, 13], methods based on sparse measurements [14-16] and hybrid methods $[17,18]$ and

c) isolating the fault by sending a crew to verify the localization of the fault and fix the problem

Due to their increased complexity and the lack of available sensors, the LV distribution grids, have not been on the center of researchers' attention, with few exceptions [7, $15,16,19]$. The vast majority of the studies focuses on the Medium Voltage (MV) distribution network. Furthermore, some of the most widely used methods such as the impedance 
based method, present some drawbacks; a typical example is the identification of multiple locations of the same distance from the feeder as possible fault locations. Finally, since high impedance faults are very difficult to detect as the fault current magnitude is very close to the one under normal operation, the vast majority of the fault localization studies examined only low impedance faults.

In order to address the above problems, a fault localization method for LV distribution grids based on an idea initially developed for MV networks [20], is proposed in this paper. Single-phase to ground SC faults were studied since they are the most frequent type of faults in a distribution grid [2]. Finally, high impedance faults with fault resistance values of up to $1000 \Omega$ were included in the study.

The paper is divided in five Sections. In the following Section the characteristics of the grid under study are presented. The fault localization method is presented in Section three. The method accuracy is analyzed in the results Section. The paper concludes with a synopsis of the achievements accomplished during this study.

\section{Grid Characteristics}

For the purpose of this study, a real case semi-rural radial LV distribution grid of Portugal was used. The connection to the MV is achieved through a distribution transformer. The grid is a three-phase-four-wire one with a solidly grounded neutral. It consists of three main feeders and a total of thirty three nodes with eighteen microgenerators (single-phase PV installations) and forty eight single-phase loads. The single line diagram of the LV distribution grid is presented in Fig. 1 along with the considered as available measurements and the fault locations under study. Two basic assumptions were made at this point: a) phase rms voltage measurements were available at each node of the grid and b) the measurements were synchronous and non erroneous.

Two are the basic characteristics of this grid that complexify its analysis: imbalance and heterogeneity. The imbalance term was used to describe the fact that load and microgenerators of different sizes are spread assymetrically throughout the grid. The per feeder and per phase connection distribution of load contracted power $(k V A)$ and microgenerator installed power $(k W)$ is presented in Table 1. At the same time, eleven different types of conductors in terms of resistance and reactance connect the nodes with each other with lengths ranging from 35 to a maximum of $210 \mathrm{~m}$ thus attributing to the grid an heterogeneous nature.

As it will be explained later, the localization method was based only on the rms voltage profile across the faulty line. Hence, the phasor mode was selected to perform the simulations in order to reduce the computational time. Furthermore, the faults were studied on their steady state, $150 \mathrm{~ms}$

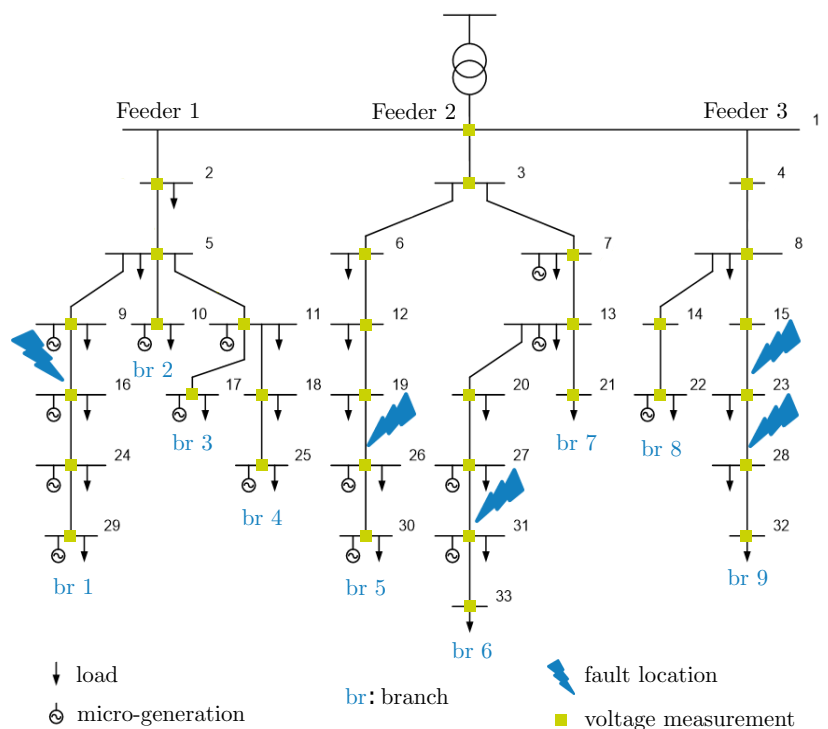

Fig. 1: Single line diagram of the LV grid with the SC fault cases. Two categories of faults are presented: a) faults close to the beginning of the feeder (green symbols) and b) faults towards the middle of the end of the feeder (blue symbols).

Table 1: Grid loads and $\mu$ gens

\begin{tabular}{c|cccccc}
\hline \hline \multirow{2}{*}{ Feeder } & \multicolumn{4}{|c}{ Contracted Power $(k V A)$} & \multicolumn{4}{c}{ Installed Power $(k W)$} \\
& $S_{a}$ & $S_{b}$ & $S_{c}$ & $P_{a}$ & $P_{b}$ & $P_{c}$ \\
\hline \hline 1 & 31.05 & 41.40 & 48.30 & 3.68 & 8.83 & 14.49 \\
2 & 3.45 & 41.40 & 24.15 & 10.58 & 7.13 & 7.08 \\
3 & 10.35 & 13.80 & 17.25 & 10.58 & 7.13 & 7.08 \\
\hline \multirow{2}{*}{ Total } & $\mathbf{7 5 . 9 0}$ & $\mathbf{9 6 . 6 0}$ & $\mathbf{8 9 . 7 0}$ & $\mathbf{1 7 . 9 4}$ & $\mathbf{1 5 . 9 6}$ & $\mathbf{2 3 . 2 7}$ \\
\hline \hline
\end{tabular}

after their occurrence. This time frame secured that the fault would appear on a steady state and before any protective device had isolated the installed microgeneration units; an action from the protective elements is expected around 200 $m s$ from the fault occurrence. This way the influence of the microgeneration participation on the voltage profile could be studied.

At this point and before proceeding to the following Section, it is necessary to introduce two notions: the branches and sectors of the grid. The branch of a grid is defined as a unique line path from the beginning of the feeder to each terminal node (e.g. first branch: from node one to node twenty nine and fifth branch: from node one to node thirty). The nine different branches are presented in Fig. 1. Moreover, the sector is defined as the section of the grid between two consecutive available measurements. In this case, since voltage measurements are available at each node, the sector is the line connecting two adjacent nodes (e.g. first sector: from 
node one to node two and second sector: from node two to node five).

\section{Fault Localization Method}

Once an alarm signal indicates the occurrence of a fault in one of the grid feeders, the fault localization process is initiated. This process is divided in three distinct steps: a) faulty branch identification, b) faulty sector localization and c) fault distance estimation. All these steps were solely based on rms voltage measurements.

\subsection{Faulty Branch Identification}

Identifying the faulty branch within a feeder with multiple branches is the first and most important step of the fault localization process. It is expected that the branch under fault would present the highest voltage drop within the faulty feeder. The branch to which the node with the lowest voltage belonged was identified and this branch was considered to be the one under fault.

\subsection{Faulty Sector Localization}

Following the faulty branch identification process, the faulty sector localization method is where the core idea of this study lies. The basic principle is based on the fact that across the line of a faulty branch, voltage is expected to drop linearly up until the faulty sector and stabilize to a certain value

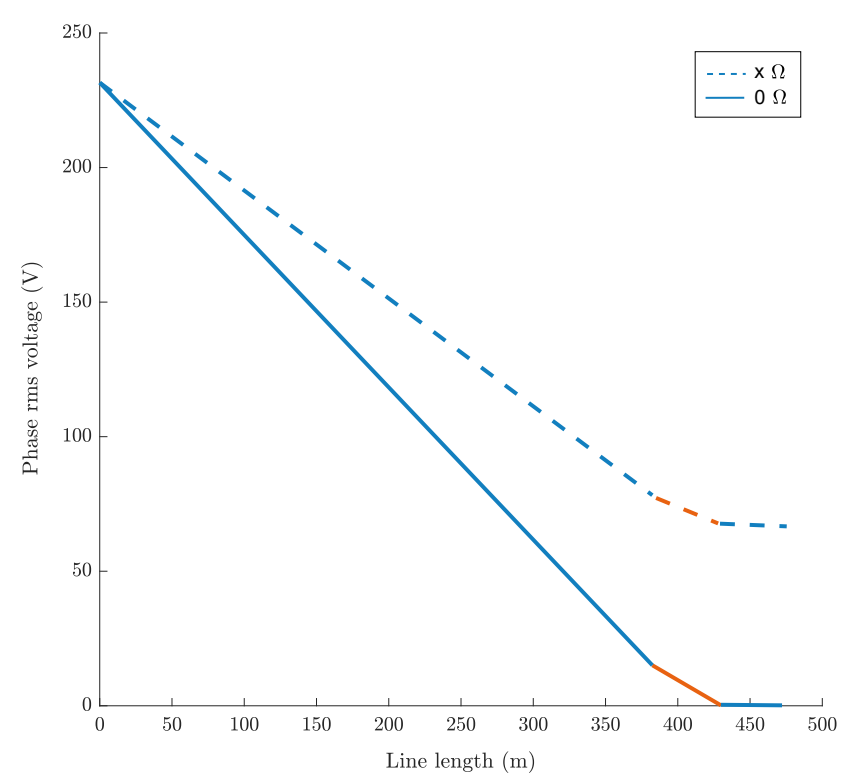

Fig. 2: Theoretical voltage profile across a faulty line for a single-phase to ground SC fault for two cases of fault resistance values: zero (" 0 ") and non-zero (" $x$ "). after the faulty sector. In Fig 2, the theoretical voltage profile and the form of its curve is presented. For $0 \Omega$ of fault resistance, the voltage will drop to zero after the faulty sector since there will be no current flowing through this part of the grid; all the current will flow from the line to the ground through their established connection during the fault leaving no circulating current in the part of the line after the fault's location. However, for any value " $x$ " of fault resistance, the voltage will stabilize to a higher value.

As mentioned in the Introduction, this idea was conceived in the context of the MV grid which is less complex than the LV grid. Although in reality the unbalanced and/or heterogeneous nature of the LV grid can affect the form of the voltage profile, the basic principle of the voltage stabilizing to a value after the faulty sector, still applies. Such an example is provided in Fig. 3 where the available measurements across the line are depicted with circles. The linear interpolation method was used to create the lines connecting these points. The change in the slope of the voltage curve is obvious as it decreases to almost zero after the faulty sector; the faulty sector is indicated by an orange line in Fig. 3.

In order to transform the above critical observation regarding the slope into an algorithm and identify the faulty sector, the two following criteria were developed:

1) if the difference between two consecutive voltage measurements was positive, signifying a change in the sign of the slope, then the previous sector was the one under fault and

2) if the absolute value of the difference between two adjacent voltage measurements was the lowest within the

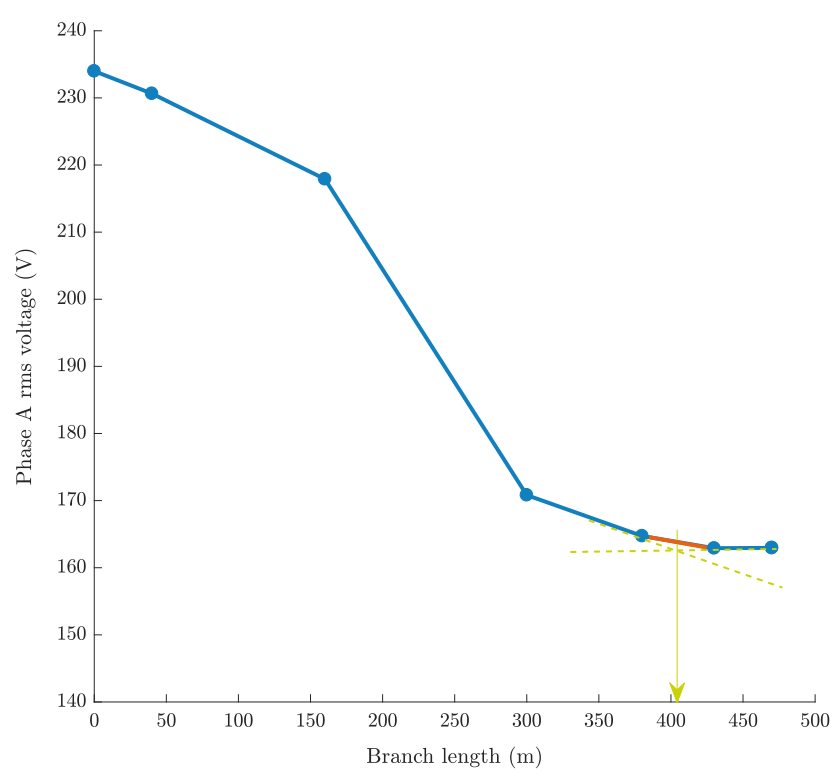

Fig. 3: Snapshot of the voltage profile along the branch length for a single-phase to ground fault (AG) between nodes 19 and 26 of a fault resistance of $1 \Omega$ at $01 h 01 \mathrm{~m} 0.15 \mathrm{~s}$. 
branch, signifying a stabilization of the curve, then the previous sector was the one under fault.

The slope of the curve is almost always negative since voltage is decreasing. However, a positive value, of the slope after the faulty sector, is possible under the presence of microgeneration units in this part of the grid thus explaining the choice of the first criterion; the effect of microgeneration units is thoroughly explained in Section V.

\subsection{Fault Distance Estimation}

The last step in localizing the fault, after identifying the faulty branch and sector, is to estimate its location within the faulty sector. To achieve that purpose, a graphic method was implemented. From the linearly interpolated curve of Fig. 3 the lines of the sectors adjacent to one under fault were linearly extrapolated (green dashed lines) and their intersection point was used to estimate the location of the fault inside the sector.

To measure the method accuracy the following formula described in [21] was used:

$\operatorname{error}(\%)=\frac{\left|d_{\text {estimated }}-d_{\text {actual }}\right|}{l_{\text {total }}} \cdot 100$

where the estimated distance is compared to the actual one and the result is normalized over the total length of the line. In Fig. 3, the fault occurs at $01 h 01 \mathrm{~m}$ with a fault resistance of $1 \Omega$ and it is located in the fifth sector of the fifth branch and at a distance of $405 \mathrm{~m}$ from the beginning of the feeder. The estimated distance for this case is $404.4 \mathrm{~m}$ presenting an accuracy of $99.38 \%$ or a deviation of $0.6 \mathrm{~m}$.

As a last step, a threshold check was applied to ensure that the estimated location falls within the limits of the identified faulty sector. This corrective process helped minimize false estimations. The results of the entire fault localization method are presented in the following Section.

\section{Results}

In order to verify the robustness of the method, the effects of the following parameters on the method were investigated. First of all, positive sequence component of the voltage was considered as an alternative to the phase rms voltage. Secondly, the effect of using conductors of different types in terms of resistance, reactance and length was examined. Furthermore, based on the load and microgeneration profiles during a given day as shown in Fig. 4, five scenarios of different combinations of load and microgeneration penetration were selected: a) at $04 \mathrm{~h}(0 \%$ load, $0 \% \mu \mathrm{gen}), \mathrm{b})$ at $01 \mathrm{~h}$

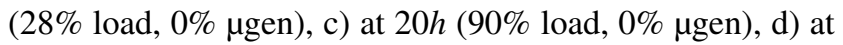
$14 h(60 \%$ load, $49 \%$ gen $)$ and e) at $12 h(50 \%$ load, $100 \%$ $\mu g$ n). Scenario (a) was used for an heterogeneity analysis, (b,c) and (d,e) to monitor how the developed method is affected by an increase of load or microgeneration respectively. Moreover, it was assumed that a maximum of $30 \%$ of the loads would operate simultaneously. Finally, the effect of increasing the fault resistance was studied by simulating faults with fault resistance values of: $0.1,1,5,10,50,100$, 500 and $1000 \Omega$. The results are discussed analytically in the following subsections.

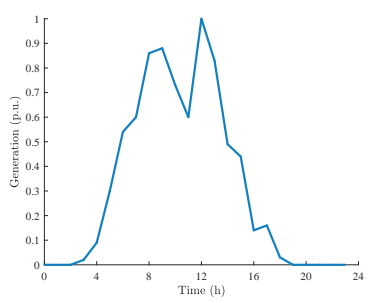

(a) Microgeneration profile

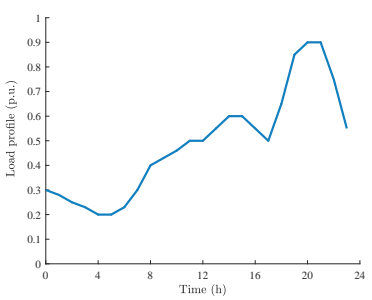

(b) Load profile
Fig. 4: Grid characteristics

\subsection{Phase Voltage vs. Positive Sequence Component}

Both the phase voltage measurements and the positive sequence component were used for all the different singlephase to ground fault cases and scenarios. In Table 2, the results for both methods are presented. It is demonstrated that the phase rms voltage measurements were more suitable for single-phase to ground faults since they presented a higher precision rate in identifying the faulty branch which is the most important step of the method. The rest of the results are presented on the basis of the phase rms voltage measurements.

Table 2: Method accuracy (\%)

\begin{tabular}{ccccc}
\hline \hline & \multicolumn{2}{c}{ Overall } & \multicolumn{2}{c}{ Less than $\mathbf{5 0} \Omega$} \\
& $\mathrm{v}_{\text {phase }}$ & $\mathrm{v}_{\text {pos }}$ & $\mathrm{v}_{\text {phase }}$ & $\mathrm{v}_{\text {pos }}$ \\
\hline \hline branch & 75.00 & 66.88 & 91.67 & 89.58 \\
sector & 58.61 & 82.87 & 81.82 & 99.53 \\
distance & 89.33 & 86.73 & 93.11 & 88.68 \\
\hline \hline
\end{tabular}

\subsection{High Impedance vs. Low Impedance Faults}

Microgeneration units are expected to contribute to the grid's voltage by increasing it while loads will cause a voltage drop. Higher impedance faults $(>50 \Omega)$ strongly affect the voltage profile bringing it very close to the normal operating conditions. In that way the effect of the microgeneration or 
the loads on the voltages profile was amplified thus enhancing the error in localizing the correct sector and estimating the fault's location.

From Table 2, it is clear that for faults of a fault resistance of less than $50 \Omega$ the precision of the method in identifying the correct branch and sector is significantly increased by $16.67 \%$ and $23.21 \%$ respectively. This means that by increasing the fault resistance, misidentification of a faulty branch and or mislocalization of a faulty sector are to be expected. An illustrative example is provided in Fig. 5.

\subsection{Imbalance Analysis}

Load and microgeneration participation, as discussed in Section 2, can affect the method since they affect the form of the voltage curve. The less elements present in the faulted branch the more accurate the method will be. Since microgeneration units increase the voltage they can, at some extent, mitigate the effect of increased load participation which tend to lower the voltage of the grid. Such an example is presented in Fig. 5. It is shown that increased load effects are more severe than the ones caused by microgeneration presence; in Fig. 5 the difference between 12 and $14 h$ when microgeneration increases from $49 \%$ to $100 \%$ is almost negligible. Especially for high impedance faults, increased penetration of either load or microgeneration units, is likely to lead to a misidentification of the faulty branch or a misslocalization of the faulty sector. The latter will cause a significant increase of the distance estimation error.

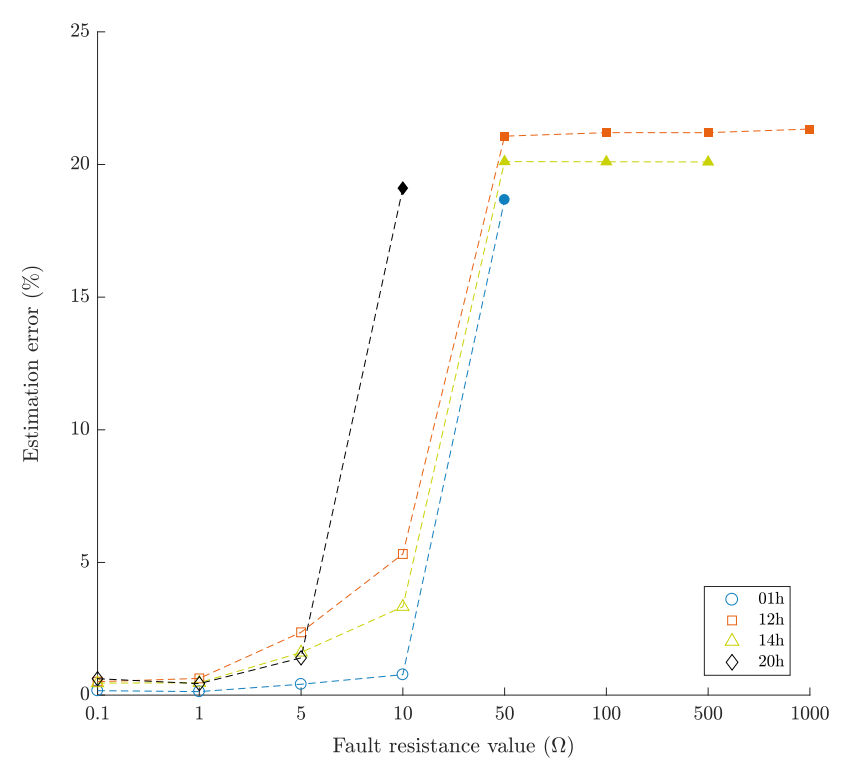

Fig. 5: Distance estimation error for a single-phase to ground fault (AG) between nodes 19 and 26. Filled markers indicate a mislocalized sector. The figure only shows results for a successful faulty branch identification.

\subsection{Heterogeneity Analysis}

A study was done without any load and microgeneration present in the grid in order to investigate the effect of the heterogeneity of the grid on the method. One parameter was set: the distance of the fault from the beginning of each feeder. Two fault cases were studied: single-phase to ground SC faults at a distance of a) $270 \mathrm{~m}$ and b) $400 \mathrm{~m}$ from the beginning of the feeder. The comparative results are presented in Table 3. Different distances were chosen since an increase of the fault distance would include a bigger variety of conductors per branch. An analysis was made for one branch per feeder; for the case of $400 \mathrm{~m}$, two branches were selected from the second feeder since the maximum length of a branch within the first feeder is $340 \mathrm{~m}$. Branch five belonging at the second feeder ( $\mathrm{f} 2$, br5) was chosen because the differences in resistance and reactance between the conductors composing it were not that important. For the exact opposite reason, branch nine of feeder three was selected (f3, br9). Additionally, branch nine is also the longest of the grid.

Two basic conclusions can be drawn from Table 3. First of all, depending on the composition of a branch and how heterogeneous it is, a cost of a maximum of almost $5 \%$ in the accuracy of the method is possible. Increasing the number of different conductors in a line also increases the the risk of loosing in precision if the conductors composing the branch are of quite different type. For example, an increase of the fault distance from $270 \mathrm{~m}$ to $400 \mathrm{~m}$ within branch nine which is quite heterogeneous would cost about $4.8 \%$ in accuracy of the estimation. Secondly, it should be underlined that since there were no loads or microgeneration units participating in this case, the increase of the fault resistance did not affect the localization method in any way. This is due to the fact that the stabilization of the curve to an exactly zero slope after the faulty sector was guaranteed.

\section{Conclusions}

A localization method for single-phase to ground SC faults in a LV distribution grid was developed in this study. Two approaches regarding the voltage measurements to be used were examined: the rms phase measurements and the positive sequence component of voltage. Different combinations of load and microgeneration participation were taken into account. Additionally, both low and high impedance faults were implemented ( $R_{f}$ from 0.1 to $1000 \Omega$ ). Finally, the effect of the heterogeneity of the grid was analyzed as well. The basic conclusions can be summarized in the following points :

1) Phase rms voltage measurements are more suitable for single-phase to ground faults than any of the symmetrical components of the voltage. 
Table 3: Distance estimation error (\%) due to heterogeneity of the grid

\begin{tabular}{ccccccc}
\hline \hline \multirow{2}{*}{$R_{f}(\Omega)$} & \multicolumn{3}{c}{$\mathbf{2 7 0} m$} & & \multicolumn{3}{c}{$\mathbf{4 0 0} m$} \\
& f1, br1 & f2, br6 & f3, br9 & f2, br5 & f2, br 6 & f3, br9 \\
\hline \hline $\mathbf{0 . 1}$ & 4.52 & 0.20 & 0.01 & 0.03 & 4.40 & 4.79 \\
$\mathbf{1}$ & 4.54 & 0.20 & 0.01 & 0.01 & 4.40 & 4.79 \\
$\mathbf{5}$ & 4.54 & 0.20 & 0.00 & 0.00 & 4.40 & 4.79 \\
$\mathbf{1 0}$ & 4.55 & 0.20 & 0.00 & 0.00 & 4.40 & 4.79 \\
$\mathbf{5 0}$ & 4.55 & 0.20 & 0.00 & 0.00 & 4.40 & 4.79 \\
$\mathbf{1 0 0}$ & 4.55 & 0.20 & 0.00 & 0.00 & 4.40 & 4.79 \\
$\mathbf{5 0 0}$ & 4.55 & 0.20 & 0.00 & 0.00 & 4.40 & 4.79 \\
$\mathbf{1 0 0 0}$ & 4.55 & 0.20 & 0.00 & 0.00 & 4.40 & 4.79 \\
\hline \hline
\end{tabular}

2) The distance estimation error increases by the increase of the fault resistance. For low impedance faults distance estimation accuracy reaches $93.11 \%$.

3) Load and microgeneration presence might lead to misidentification of the faulty branch or mislocalization of the faulty sector decreasing the precision of the method with load effects being more severe than the ones caused by microgeneration.

4) The use of different type of conductors in the studied LV grid may increase the distance estimation error up to $5 \%$.

Acknowledgements This project has received funding from the European Union's Horizon 2020 research and innovation programme under the Marie Skłodowska-Curie grant agreement No 675318 (INCITE).

\section{References}

1. P. Linares and L. Rey, "The costs of electricity interruptions in Spain. Are we sending the right signals?" Energy Policy, vol. 61, pp. 751-760, Oct. 2013.

2. T. Gönen and T. Gönen, Electric Power Distribution Engineering. Boca Raton, FL: CRC Press, 2014, oCLC: 879683602.

3. CEER, "6th Benchmarking Report on the Continuity of Electricity and Gas Supply," Brussels, Tech. Rep. C 18 - EQS - 86 - 03, 2016.

4. A. Shivakumar, M. Welsch, C. Taliotis, D. Jakšić, T. Baričević, M. Howells, S. Gupta, and H. Rogner, "Valuing blackouts and lost leisure: Estimating electricity interruption costs for households across the European Union," Energy Research \& Social Science, vol. 34, pp. 39-48, Dec. 2017.

5. A. Bahmanyar, S. Jamali, A. Estebsari, and E. Bompard, "A comparison framework for distribution system outage and fault location methods," Electric Power Systems Research, vol. 145, pp. 1934, Apr. 2017.

6. R. Dashti, M. Ghasemi, and M. Daisy, "Fault location in power distribution network with presence of distributed generation resources using impedance based method and applying $\pi$ line model," Energy, vol. 159, pp. 344-360, Sep. 2018.
7. F. C. L. Trindade and W. Freitas, "Low Voltage Zones to Support Fault Location in Distribution Systems With Smart Meters," IEEE Transactions on Smart Grid, vol. 8, no. 6, pp. 2765-2774, Nov. 2017.

8. C. Orozco-Henao, A. S. Bretas, A. R. Herrera-Orozco, J. D. Pulgarín-Rivera, S. Dhulipala, and S. Wang, "Towards active distribution networks fault location: Contributions considering DER analytical models and local measurements," International Journal of Electrical Power \& Energy Systems, vol. 99, pp. 454-464, Jul. 2018.

9. M. Majidi, M. Etezadi-Amoli, and M. S. Fadali, "A Novel Method for Single and Simultaneous Fault Location in Distribution Networks," IEEE Transactions on Power Systems, vol. 30, no. 6, pp. 3368-3376, Nov. 2015.

10. Y. Dong, C. Zheng, and M. Kezunovic, "Enhancing Accuracy While Reducing Computation Complexity for Voltage-Sag-Based Distribution Fault Location," IEEE Transactions on Power Delivery, vol. 28, no. 2, pp. 1202-1212, Apr. 2013.

11. M. Sarvi, "Determination of Fault Location and Type in Distribution Systems using Clark Transformation and Neural Network," International Journal of Applied Power Engineering (IJAPE), vol. 1, no. 2, Aug. 2012.

12. A. Borghetti, M. Bosetti, C. A. Nucci, M. Paolone, and A. Abur, "Integrated Use of Time-Frequency Wavelet Decompositions for Fault Location in Distribution Networks: Theory and Experimental Validation," IEEE Transactions on Power Delivery, vol. 25, no. 4, pp. 3139-3146, Oct. 2010.

13. H. Nouri, C. Wang, and T. Davies, "An accurate fault location technique for distribution lines with tapped loads using wavelet transform," in 2001 IEEE Porto Power Tech Proceedings (Cat. No.01EX502), vol. 3, Sep. 2001, pp. 4 pp. vol.3-.

14. M. Majidi, A. Arabali, and M. Etezadi-Amoli, "Fault Location in Distribution Networks by Compressive Sensing," IEEE Transactions on Power Delivery, vol. 30, no. 4, pp. 1761-1769, Aug. 2015.

15. N. Silva, F. Basadre, P. Rodrigues, M. S. Nunes, A. Grilo, A. Casaca, F. Melo, and L. Gaspar, "Fault detection and location in Low Voltage grids based on distributed monitoring," in 2016 IEEE International Energy Conference (ENERGYCON), Apr. 2016, pp. $1-6$.

16. L. Marques, N. Silva, I. Miranda, E. Rodriges, and H. Leite, "Detection and localisation of non-technical losses in low voltage distribution networks," in Mediterranean Conference on Power Generation, Transmission, Distribution and Energy Conversion (MedPower 2016), Nov. 2016, pp. 1-8.

17. A. C. Adewole, R. Tzoneva, and S. Behardien, "Distribution network fault section identification and fault location using wavelet entropy and neural networks," Applied Soft Computing, vol. 46, pp. 296-306, Sep. 2016.

18. T. Gush, S. B. A. Bukhari, R. Haider, S. Admasie, Y.-S. Oh, G.-J. Cho, and C.-H. Kim, "Fault detection and location in a microgrid using mathematical morphology and recursive least square methods," International Journal of Electrical Power \& Energy Systems, vol. 102, pp. 324-331, Nov. 2018.

19. G. Niu, L. Zhou, W. Pei, and Z. Qi, "A novel fault location and recognition method for low voltage active distribution network," in 2015 5th International Conference on Electric Utility Deregulation and Restructuring and Power Technologies (DRPT), Nov. 2015, pp. 876-881.

20. A. Teninge, C. Pajot, B. Raison, and D. Picault, "Voltage profile analysis for fault distance estimation in distribution network," in 2015 IEEE Eindhoven PowerTech, Jun. 2015, pp. 1-5.

21. M. M. Saha, J. Izykowski, and E. Rosołowski, Fault Location on Power Networks, ser. Power systems. London: Springer, 2010, oCLC: ocn495597133. 